\title{
Thermal Gating of Magnon Exchange in Magnetic Multilayers with Antiferromagnetic Spacers
}

\author{
D. M. Polishchuk®, ${ }^{1,2, *}$ Yu. O. Tykhonenko-Polishchuk, ${ }^{1,2}$ Ya. M. Lytvynenko, ${ }^{2}$ \\ A. M. Rostas, ${ }^{3}$ O. V. Gomonay, ${ }^{4}$ and V. Korenivski ${ }^{1}$ \\ ${ }^{1}$ Nanostructure Physics, Royal Institute of Technology, 10691 Stockholm, Sweden \\ ${ }^{2}$ Institute of Magnetism of the NAS of Ukraine and MES of Ukraine, 03142 Kyiv, Ukraine \\ ${ }^{3}$ National Institute of Materials Physics, 077125 Bucharest-Magurele, Romania \\ ${ }^{4}$ Institut für Physik, Johannes Gutenberg Universität Mainz, D-55099 Mainz, Germany
}

(Received 22 October 2020; revised 5 March 2021; accepted 5 May 2021; published 4 June 2021)

\begin{abstract}
We observe a strong thermally controlled magnon-mediated interlayer coupling of two ferromagnetic layers via an antiferromagnetic spacer in spin-valve type trilayers. The effect manifests itself as a coherent switching as well as collective resonant precession of the two ferromagnets, which can be controlled by varying temperature and the spacer thickness. We explain the observed behavior as due to a strong hybridization of the ferro- and antiferromagnetic magnon modes in the trilayer at temperatures just below the Néel temperature of the antiferromagnetic spacer.
\end{abstract}

DOI: 10.1103/PhysRevLett.126.227203

Introduction.-Interlayer exchange coupling in magnetic multilayers is one of the key functional properties for spinelectronic applications [1-3]. Ferromagnetic interlayer exchange can lead to perpendicular magnetic anisotropy used in ultrahigh-density storage media [4-6]. The exchange bias found in ferromagnetic-antiferromagnetic (F-AFM) bilayers is commonly exploited in spin-valve sensors [7-9]. Indirect interlayer exchange enables the giant magnetoresistive superlattices [10-12] and synthetic antiferromagnets $[13,14]$. Interlayer exchange through antiferromagnetic spacers in F-AFM-F trilayers $[15,16]$ makes AFM materials attractive as mediators of interlayer coupling in nanostructures [17-22], which is of special interest for the rapidly developing field of antiferromagnetic spintronics $[13,23]$ and has the potential to enable ultrafast (THz), compact, and highly stable to disturbing magnetic field nanodevices.

Postfabrication control of the interlayer exchange in a magnetic multilayer can yield additional functionality. For example, thermal control is employed in thermally assisted magnetic memory [24] and is also promising for developing spin-thermionic valves and oscillators [25-29]. Such devices often use the Curie transition in a weakly ferromagnetic spacer as the interlayer coupling mechanism. An interesting fundamental question is whether an antiferromagnetic spacer can be used as the controlling element for

Published by the American Physical Society under the terms of the Creative Commons Attribution 4.0 International license. Further distribution of this work must maintain attribution to the author(s) and the published article's title, journal citation, and DOI. Funded by Bibsam. on-off switching of interlayer exchange. A successful demonstration could enable a variety of applications in systems where wide-frequency-range oscillators are used, including the presently intensively discussed spinoscillator-based neuromorphic circuits [30].

In this Letter, we demonstrate a method for manipulating coupling of thin ferromagnetic layers separated by an antiferromagnetic spacer in F-AFM-F trilayers using thermal gating of the magnetic state of the spacer. We use layer-selective magneto-optical Kerr-effect (MOKE) and ferromagnetic resonance (FMR) measurements, enabled by having the $\mathrm{F}$ layers of different magnetic anisotropy (soft $\mathrm{F}$ and hard $\mathrm{F}^{*}$ ), in order to reconstruct the thermomagnetic state diagram of the trilayers and identify the regions of either coupled or independent field switching and resonant precession of the $\mathrm{F}$ layers. We associate the coupled regime with a strong hybridization of the ferro- and antiferromagnon modes, which establishes correlations between the ferromagnetic layers. Such correlations are strongly enhanced in the vicinity of the Néel transition temperature due to the softening of the antiferromagnetic magnons in this range and can be efficiently switched on and off by varying the sample temperature. The demonstrated ex situ control of interlayer coupling in magnetic nanostructures via thermal gating of antiferromagnetic magnons near room temperature is promising for spintronic applications.

Theory.-Recently, Cheng et al. [31,32] analyzed the magnon-mediated coupling in F-AFM-F trilayers by considering excitations in the AFM layer only. Here we develop a model that has at its core hybridization of ferro- and antiferromagnetic magnon modes, focusing on the switching conditions for the $\mathrm{F}$ and $\mathrm{F}^{*}$ layers in the $\mathrm{F}^{*}$-AFM-F trilayers. Assuming the switching is thermally assisted, we 
search for nonlocalized magnon modes able to correlate thermal fluctuations in $\mathrm{F}$ and $\mathrm{F}^{*}$ [33]. Our approach is based on associating $\mathrm{F}$ switching with softening of the lowest magnon mode whose frequency $\omega_{\mathrm{F}} \propto \sqrt{H_{\mathrm{cr}}-H} \rightarrow 0$ at some characteristic critical field, $H=H_{\mathrm{cr}}$. We model the spacer as a collinear uniaxial antiferromagnet [34], whose magnetic order is characterised by Néel vector $\mathbf{N}$, and assume parallel alignment of the easy axes in the $\mathrm{F}$ and AFM layers-the typical experimental layout. We further assume that the exchange coupling at the $\mathrm{F}^{*}-\mathrm{AFM}$ and AFM-F interfaces, parametrized by constants $H_{b}^{*}$ and $H_{b}$, favors parallel alignment of all the magnetic vectors. Magnons are considered as small excitations $\delta \mathbf{M}^{*}, \delta \mathbf{M}$, and $\delta \mathbf{N}$ over the equilibrium state, in which $\mathbf{M}^{*} \uparrow \uparrow \mathbf{M} \uparrow \uparrow \mathbf{N}$. Thermal effects manifest via the temperature dependent AFM parameters, such as the sublattice magnetizations, $|\mathbf{N}| / 2$, and the AFM resonance frequency, $\omega_{\mathrm{AFM}}$, all vanishing at the Néel temperature, $T_{N}$; $|\mathbf{N}|, \omega_{\mathrm{AFM}} \propto \sqrt{T_{N}-T}$.

Figure 1 shows the temperature dependence of the $\mathrm{F}^{*}$-AFM-F magnon modes calculated in the limit of a thin AFM layer $(t \rightarrow 0)$. Predictably, in the vicinity of the avoided crossing, $\omega_{\mathrm{AFM}}\left(T_{1}\right)=\omega_{\mathrm{F}^{*}} \quad\left[\omega_{\mathrm{AFM}}\left(T_{2}\right)=\omega_{\mathrm{F}}\right]$, oscillations of the Néel vector are strongly hybridized with the oscillations in the $\mathrm{F}^{*}$ and $\mathrm{F}$ layers, as shown by the relative values of $\delta M^{*}$ and $\delta M$ in each of the modes in Figs. 1(c) and 1(d). In addition, in the interval $T_{1}<T<T_{2}$ between the two crossing points, one of the modes (red line) is delocalized across all three magnetic layers and has comparable amplitudes of $\delta M^{*}$ and $\delta M$ in $\mathrm{F}$ and $\mathrm{F}^{*}$. This

(a)
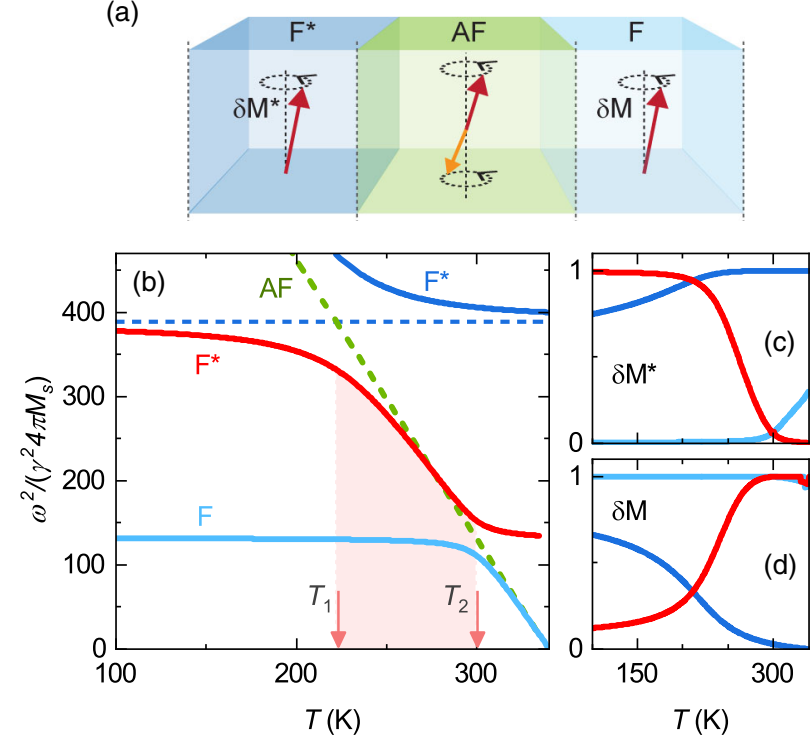

FIG. 1. (a) $\mathrm{F}^{*}$-AFM-F system schematic. (b) Magnon spectra; temperature dependence of uncoupled (dashed) and coupled (solid) magnon modes. Magnon amplitudes in $\mathrm{F}^{*}\left[\delta M^{*}\right.$; (c)] and $\mathrm{F}\left[\delta M\right.$; (d)] layers for each mode; $\mathrm{F}-\mathrm{F}^{*}$ correlations are mediated by two magnon modes that have nonzero amplitude in both $\mathrm{F}$ layers simultaneously (red lines). strongly hybridized mode produces correlations between $\mathrm{F}$ and $\mathrm{F}^{*}$ and can lead to coherent rotation and even switching of the two layers. To clarify this further, we consider the spin dynamics of the $\mathrm{F}^{*}$-AFM-F trilayer in the vicinity of the critical field $H \rightarrow H_{\text {cr. }}$. In this region the mode with $\omega=\omega_{\mathrm{F}} \rightarrow 0$, which is localized mainly in the $\mathrm{F}$ layer (light blue line), becomes unstable and amplitude $\delta M$ grows exponentially. However, due to the nonlinear coupling with the delocalized mode, the nominally localized mode stimulates exponential growth of $\delta M^{*}$ in $\mathrm{F}^{*}$, thus inducing coherent switching in the trilayer [35]. In this case the magnons in the AFM spacer play the role of a mediator, which couples $\mathrm{F}$ and $\mathrm{F}^{*}$.

Next, we discuss the effect of the AFM layer thickness, $t$, on the coupling between the $\mathrm{F}$ and $\mathrm{F}^{*}$ layers. The magnon modes in the trilayer are fully delocalized only if their frequency $\omega>\omega_{\mathrm{AFM}}, \omega_{\mathrm{F}}$, and $\omega_{\mathrm{F}^{*}}$. In contrast, the amplitude of the modes with $\omega<\omega_{\text {AFM }}$ decays exponentially within the AFM layer as $\delta M \propto \delta M^{*} \exp \left[-t \sqrt{\omega_{\mathrm{AFM}}^{2}-\omega^{2}} / c\right]$, where $c$ is the magnon velocity in the AFM layer. At intermediate temperatures, $T_{1}<T<T_{2}$, the delocalization of the modes and the resulting collective dynamics are still possible, although with a diminished effective coupling constant, $H_{b}^{*} \rightarrow H_{b}^{*} \exp \left(-t / x_{0}\right)$. The characteristic length scale $x_{0}$ depends on temperature and the interface properties.

Finally, we consider the switching process outside the inner-crossing range ( $T<T_{1}$ and $\left.T>T_{2}\right)$. At low temperature, $T<T_{1}$, the AFM magnons are hybridized mainly with the $\mathrm{F}^{*}$ magnons, since the AFM layer becomes too hard for the long-wavelength $F$ magnons $\left(\omega_{\mathrm{AFM}} \gg \omega_{\mathrm{F}}\right)$. Although the $\mathrm{F}$ magnons with the nonzero wave vectors $\mathbf{k}$, satisfying $\omega_{\mathrm{F}}(\mathbf{k}) \approx \omega_{\mathrm{AFM}}$, can still hybridize with the AFM magnons, their population is vanishingly low, defined by the temperature factor $\propto \exp \left\{-\hbar \omega_{\mathrm{AFM}} / k_{B} T\right\}$, where $\hbar$ and $k_{B}$ are the Planck and Boltzmann constants, respectively. As a result, at low temperature, most of the magnon modes are localized and each of the ferromagnetic layers switches independently. At high temperature, $T>T_{2}$, the AFM layer is soft $\left(\omega_{\mathrm{AFM}} \leq \omega_{\mathrm{F}}<\omega_{\mathrm{F}^{*}}\right)$ and transparent for the $\mathrm{F}$ and $\mathrm{F}^{*}$ magnons. In this limit, however, the effective coupling at the F-AFM and AFM-F* interfaces is significantly weakened due to the thermal fluctuations of the AFM order and the reduced value of the Néel vector $|\mathbf{N}| \rightarrow 0$. As a result, correlations between the ferromagnetic layers vanish also at high temperature.

From the above analysis we can conclude that an AFM spacer can effectively mediate coupling between two ferromagnetic layers in a finite temperature range near $T_{N}$, in which the AFM spacer is soft enough to transmit the $\mathrm{F}$ and $\mathrm{F}^{*}$ magnons and thermal fluctuations are still too weak to suppress exchange interactions at the F-AFM and AFM-F* interfaces. This opens a way of controlling switching of the ferromagnetic layers by varying the temperature. 

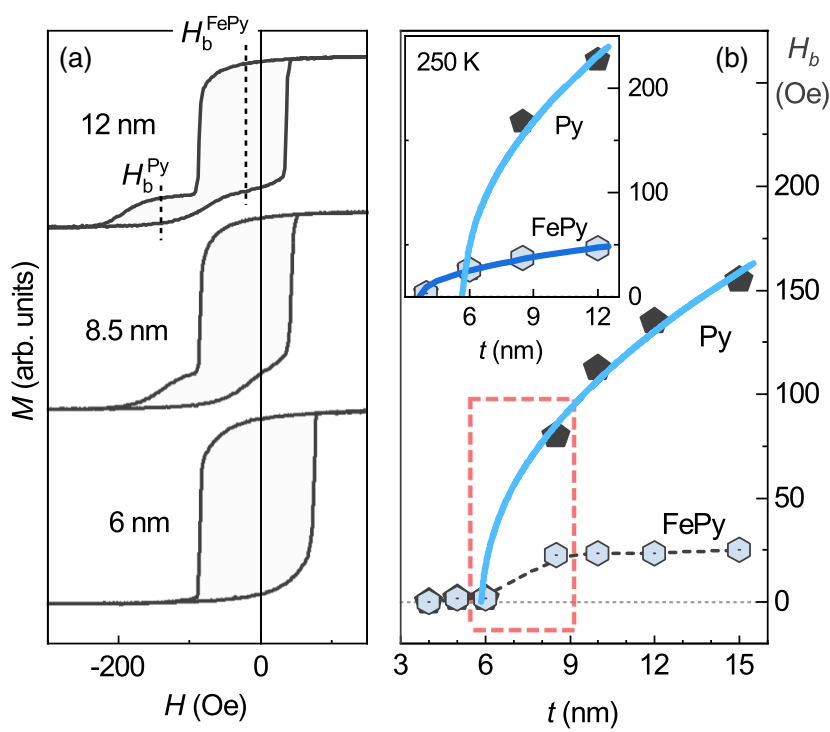

FIG. 2. Interface exchange coupling in FePy-FeMn $(t)-\mathrm{Py}$ trilayers as a function of FeMn thickness $(t)$ at $300 \mathrm{~K}$. (a) VSM magnetization loops for select AFM thicknesses.

(b) Thickness dependence of exchange bias field (loop offset) for Py and FePy $\left(H_{b}^{\mathrm{Py}}\right.$ and $\left.H_{b}^{\mathrm{FePy}}\right)$ determined as shown in (a); inset shows $H_{b}$ fields measured at $250 \mathrm{~K}$ using MOKE. Solid lines show fitting with power law $H_{b} \propto\left(t / t_{b}-1\right)^{1 / 2}$. Red dashed line encloses region with pronounced interlayer coupling.

Samples and VSM measurements.-We study $\mathrm{F}^{*}$-AFM-F trilayers of composition $[\mathrm{Fe}(6)-\mathrm{Py}(3)]-\mathrm{FeMn}(t)-\mathrm{Py}(5)$, where the layer thicknesses in nanometers are given in parentheses and $\mathrm{FeMn}$ and $\mathrm{Py}$ stand for $\mathrm{Fe}_{50} \mathrm{Mn}_{50}$ and $\mathrm{Ni}_{81} \mathrm{Fe}_{19}$. The $\mathrm{Py}(5)$ layer and the $[\mathrm{Fe}(6)-\mathrm{Py}(3)]$ bilayer are the soft and hard ferromagnetic layers, respectively (hereafter Py and FePy). The Py(3) sublayer is used to promote the growth of the FeMn layer with the desirable AFM properties $[40,41]$ as well as to make the two F-AFM interfaces compositionally identical. Since the properties of an antiferromagnet strongly depend on finite-size effects [42], we fabricated a series of trilayers with different thicknesses of the FeMn spacer $(t=4-15 \mathrm{~nm})$. All films were deposited by dc magnetron sputtering (AJA-Orion) at room temperature. In order to induce a preferred magnetization direction, the samples were deposited and subsequently annealed at $250{ }^{\circ} \mathrm{C}$ in a saturating magnetic field.

Figure 2(a) shows typical magnetization loops $(M-H)$ for the trilayers measured using vibrating sample magnetometry (VSM) at room temperature. For $t>6 \mathrm{~nm}$, the $M-H$ loops are composed of two overlapping field-offset loops that correspond to the hard FePy $\left(H_{b}^{\mathrm{FePy}}\right)$ and soft Py $\left(H_{b}^{\mathrm{Py}}\right)$ layers, respectively. The offset vanishes with decreasing $t$, which is due to the known effect of weakening AFM order in progressively thinner AFM films [9]. A field-centered, single $M-H$ loop observed for $t \leq 6 \mathrm{~nm}$ therefore indicates a vanishing exchange bias and parallel alignment of the outer ferromagnets. Thick AFM spacers $(t>10 \mathrm{~nm})$ yield essentially fully decoupled $\mathrm{F}$ and $\mathrm{F}^{*}$, where the trilayer $M-H$ loop is a superposition of two minor loops with the coercivity and field offset corresponding to FePy and Py, individually [43].

Layer-selective MOKE.-We use a photoelastic modulation (PEM) technique [36-39] for measuring the longitudinal MOKE of the samples, which distinguishes between the sample's total magnetic response [first harmonic at $50 \mathrm{kHz}$; yellow lines in Fig. 3(a)] and the magnetic response of the hard Fe-Py bilayer (second harmonic at $100 \mathrm{kHz}$; blue lines). This MOKE selectivity was calibrated against VSM measurements on the trilayers [35], which can distinguish the individual switching of the Py and FePy layers by the respective coercive fields as well as the magnetization step heights (Py and FePy were purposefully made with clearly different magnetic moments, about 1 to 3 ).

The temperature evolution of the in-plane MOKE $M-H$ loops for the FePy-FeMn(6)-Py trilayer $(t=6 \mathrm{~nm})$ is shown in Fig. 3(a). At low temperature $(T<220 \mathrm{~K})$, well below the effective $T_{N} \approx 340 \mathrm{~K}$ of the FeMn(6) spacer, the major loop consists of two superposed but clearly distinguishable minor loops, which correspond to Py and FePy. The two minor loops are offset in field due to exchange pinning by the AFM spacer. The nonequal field offsets indicate that Py and FePy are pinned individually, i.e., the

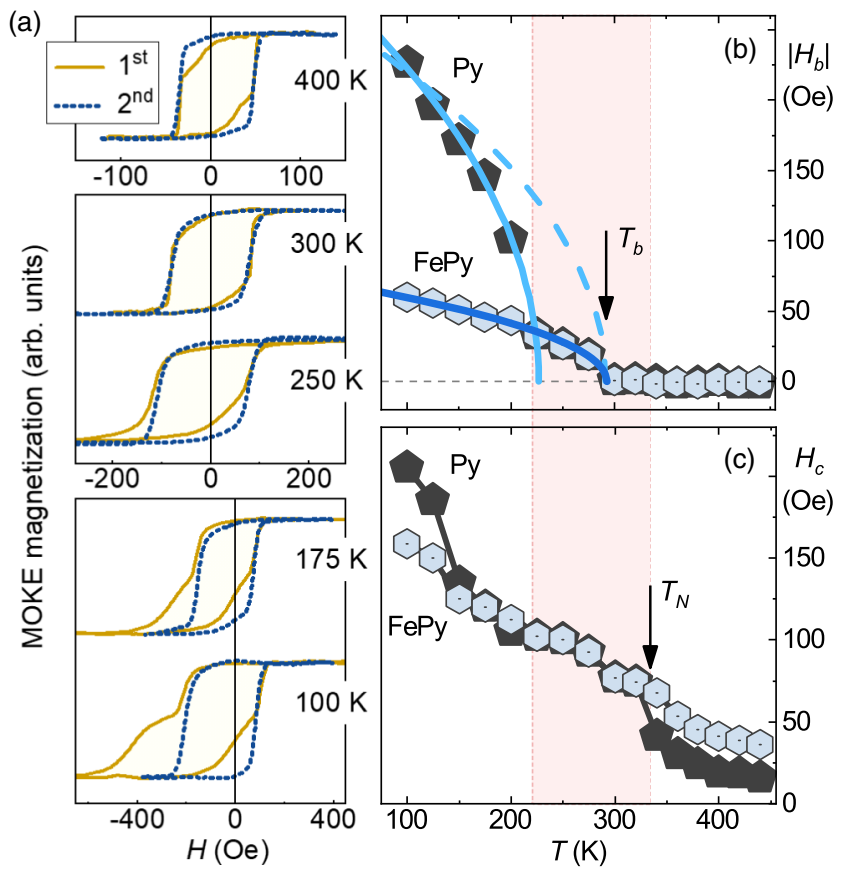

FIG. 3. Thermomagnetic transition in FePy-FeMn(6 nm)-Py. (a) MOKE loops measured at first and second harmonics of PEM frequency. Temperature dependence of exchange-bias field $H_{b}$ (b) and coercive field $H_{c}$ (c) for Py and FePy. Solid lines in (b) are fits using power law $H_{b} \propto\left(1-T / T_{b}\right)^{1 / 2}$; dashed line is behavior expected for stand-alone Py-FeMn bilayer. Shaded is region of significant interlayer coupling. 
interlayer coupling does not propagate through the 6-nmthick AFM spacer at low temperatures where the AFM order is strong. With increasing temperature toward $T_{N}$, the two minor loops in the combined $M-H$ loop (yellow, first harmonic) merge into a single loop at near room temperature, indicating that the outer $\mathrm{F}$ layers are strongly coupled through the AFM spacer. Above $T_{N}$, the single loop transforms again into a double loop (first harmonic at $400 \mathrm{~K}$ ), which is now centered at zero field. This double loop consists of two minor loops of different coercivity. As expected, the two coercive fields are equal to the intrinsic $H_{c}$ of Py and FePy, which indicates that the ferromagnets are fully decoupled and exchange unpinned when the FeMn spacer is paramagnetic.

Ferromagnetic resonance.-We have measured and compared the FMR spectra of two samples, with and without AFM-mediated interlayer exchange, having the AFM spacers 6 and $9.5 \mathrm{~nm}$ thick, respectively. Figure 4(a) illustrates the double FMR in the decoupled state of the $6 \mathrm{~nm}$ sample, from which the individual resonance fields for the Py and FePy layers can be obtained. The resonance lines merge on lowering the temperature below $T_{N}$, as
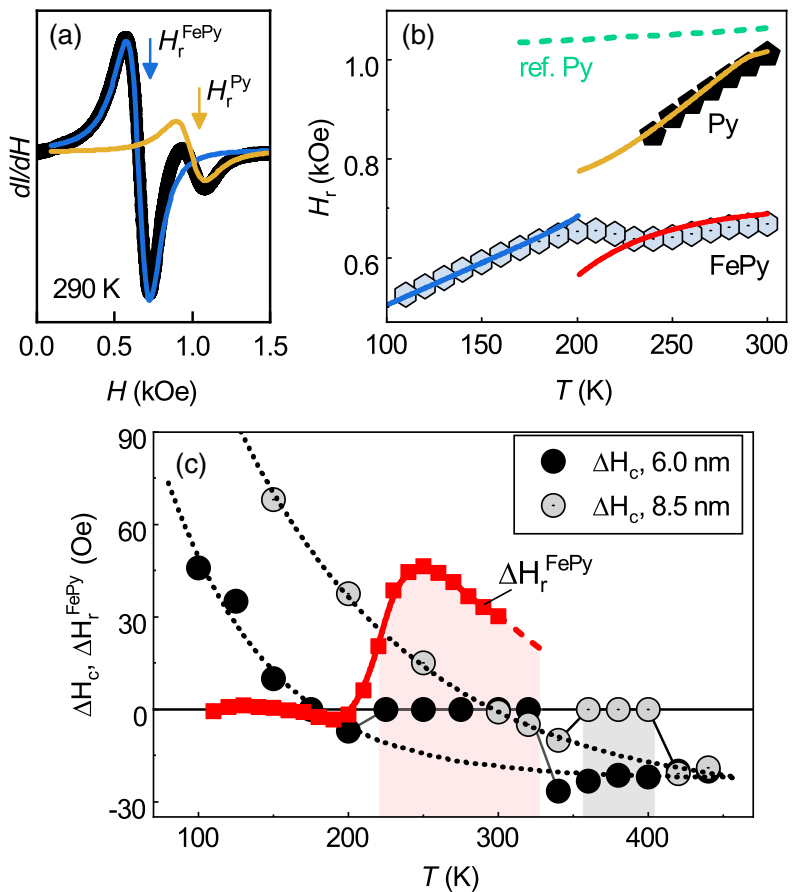

FIG. 4. (a) FMR spectrum of $6 \mathrm{~nm}$ sample recorded at $290 \mathrm{~K}$ showing two distinct peaks for Py and FePy; blue and yellow lines show model fitting for determining respective resonance fields. (b) Resonance fields of FePy and Py; green dashed line is resonance field of reference single-layer Py; solid lines are fitted theoretical magnon modes. (c) MOKE-measured coercive-field difference vs temperature for samples with 6.0 and $8.5 \mathrm{~nm}$ thick FeMn; dotted lines are guides to the eye; shaded are regions of significant interlayer coupling where $\Delta H_{c}=0$ for 6 and $8.5 \mathrm{~nm}$ samples; FMR-deduced interlayer coupling field for $6 \mathrm{~nm}$ sample [35] is shown in red. shown in Fig. 4(b), with the FePy line staying strong in intensity through the entire coupling range, thus offering a sensitive probe of the interlayer coupling strength [35]. We associate the observed merging of the Py and FePy FMR lines with the delocalized magnon mode, which is strong just below $T_{N}$ and establishes correlations between the ferromagnetic layers, in good agreement with the theory. The predicted magnon hybridization (see Theory and Fig. 1) provides excellent fits to the measured $H_{r}(T)$ of Fig. 4(b). A comparison of the temperature dependence of the FePy resonance field for the two samples [35] yields a clear differential peak, $\Delta H_{r}^{\mathrm{FePy}}$, due to the interlayer coupling at just below $T_{N}$, shown in red in Fig. 4(c). This FMR-deduced peak in the interlayer exchange coupling (IEC) shows an excellent agreement with the IEC deduced from MOKE magnetometry, both pronounced around $250 \mathrm{~K}$ [red line and region in Fig. 4(c)].

Magnetic state diagram.-The observed three magnetization regimes in the trilayer represent its thermomagnetic state diagram and are well illustrated by the temperature dependence of the exchange bias and coercive fields; Figs. 3(b) and 3(c). In particular, $H_{b}(T)$ clearly shows the low- $T$ regime with individually exchange-biased Py and FePy and, therefore, negligible ferromagnetic coupling between $\mathrm{F}$ and $\mathrm{F}^{*}$. On the other hand, the $H_{c}(T)$ dependence is very informative in characterizing the high- $T$ regime, above the Néel point of the spacer, where Py and FePy switch at their intrinsic coercive fields, again indicating negligible coupling between $\mathrm{F}$ and $\mathrm{F}^{*}$. The $H_{b}$ and $H_{c}$ values exactly overlap in the intermediate- $T$ range, just below $T_{N}$, indicating that the outer ferromagnets are fully coupled in a parallel magnetization state and show coherent switching.

The transition between the three thermomagnetic regimes is most clearly visualized by the temperature dependence of the coercive field difference $\Delta H_{c}=$ $\left(H_{c}^{\mathrm{Py}}-H_{c}^{\mathrm{FePy}}\right)$, shown in Fig. 4(c). $\Delta H_{c}$ is nonzero at low $T$ and high $T$, which indicates negligible ferromagnetic coupling between $\mathrm{F}$ and $\mathrm{F}^{*}$. In the intermediate temperature range, $\Delta H_{c}$ strictly vanishes (e.g., solid circles for the $6 \mathrm{~nm}$ sample between 220 and $320 \mathrm{~K}$ ), indicating ferromagnetically coupled $\mathrm{F}$ and $\mathrm{F}^{*}$. This conclusion from the MOKE data is strongly supported by the FMR results discussed above, which show a clear peak in the interlayer coupling in the same temperature range.

The coercivity of the Py layer is larger than that of the FePy layer at low temperatures [positive $\Delta H_{c}$ in Fig. 4(c)] since the effect of the AFM-surface scales inversely with the $\mathrm{F}\left(\mathrm{F}^{*}\right)$ layer's magnetic moment and the Py layer is both thinner and weaker magnetically. As the temperature is increased toward $T_{N}$, the two coercive fields merge $\left(\Delta H_{c}=0\right)$ due to the AFM-mediated ferromagnetic coupling. On further temperature increase, $\Delta H_{c}$ becomes negative as expected (stand-alone $\mathrm{Py}$ is softer than $\mathrm{Fe}$ ) and is the same in magnitude for the structures with 
different AFM thickness (6.0 and $8.5 \mathrm{~nm}$ ), which additionally indicates that the spacer becomes paramagnetic so $\mathrm{F}$ and $\mathrm{F}^{*}$ fully decouple. The point of decoupling (loss of AFM order at $T_{N}$ ) for the two structures is found to be, respectively $340 \mathrm{~K}$ and $410 \mathrm{~K}$, which agrees well with the known values for thin-film FeMn [42].

Importantly, the magnon-mediated correlations strongly affect the blocking temperature of the $\mathrm{F}$ and $\mathrm{F}^{*}$ layers, at which the AFM bias vanishes $\left(H_{b}=0\right)$. We determine the blocking temperature $T_{b}$ by fitting $H_{b}(T)$ to a power law consistent with the Landau phase transition theory $\left[\propto\left(1-T / T_{b}\right)^{1 / 2}\right.$; see Fig. 3(b)]. While the individual bilayers Py-FeMn and FePy-FeMn have identical interfaces and thus should have the same $T_{b}$ [vertical arrow in Fig. 3(b)], the trilayer data show a large drop in the effective blocking temperature of the soft layer, $T_{b}^{\text {soft }}<T_{b}$. This drop (from about 300 to $220 \mathrm{~K}$ ) is a direct effect of the enhanced fluctuations in the soft layer due to the nonlocalized magnon modes excited in the hard layer. In contrast, and in line with expectations, the hard layer is much less sensitive to the influence of the soft layer's magnons (its effective $T_{b}^{\text {hard }} \approx T_{b}$ ) and, therefore, defines the blocking temperature of the trilayer. As $T_{b}^{\text {hard }}$ is found midway the region of strong interlayer correlations (bound by $T_{b}^{\text {soft }}$ and $T_{N}$ ) the coherent switching is, in fact, found to take place with $\left(T_{b}^{\text {soft }}<T<T_{b}^{\text {hard }}\right)$ or without $\left(T_{b}^{\text {hard }}<T<T_{N}\right)$ exchange bias. Remarkably, the temperature and AFM-thickness dependence of the observed interlayer coupling are complementary, which becomes evident upon comparing Fig. 3(b) with Fig. 2(b), where $H_{b}(t)$ and $H_{b}(T)$ show the same power law dependence.

Conclusions. - The fact that $\mathrm{F}^{*}$-AFM-F trilayers are ferromagnetically coupled at temperatures just below $T_{N}$ of the AFM spacer indicates a thermomagnetic origin of the effect. At $T \lesssim T_{N}$, the antiferromagnet experiences strong thermal agitation resulting in a softening of its magnon modes toward the low-GHz range, which is characteristic of magnons in the ferromagnets. The low-frequency magnons can then propagate through the spacer, exchanging spin between the outer ferromagnets. At significantly lower temperatures $\left(T \ll T_{N}\right)$, the magnon gap in the AFM spacer broadens toward the $\mathrm{THz}$ range and acts as a potential barrier to interlayer magnon exchange. At high temperatures $\left(T>T_{N}\right)$, the spacer is fully spin disordered and unable to transmit magnons. Thus, from the viewpoint of interlayer $\mathrm{F}^{-\mathrm{F}^{*}}$ exchange, the AFM spacer undergoes a thermal transition of type insulator-conductor-insulator, reflected in the three distinct thermomagnetic regimes found in the system.

Support from the Swedish Research Council (201803526) and Olle Engkvist Foundation (2020:207-0460) are gratefully acknowledged. Y. L. acknowledges support from the Central European Research Infrastructure (CERIC) Consortium (Horizon 2020, project
ACCELERATE, No. 731112). O. G. acknowledges support from the Alexander von Humboldt Foundation, the ERC Synergy Grant SC2 (No. 610115), and the Deutsche Forschungsgemeinschaft (DFG, German Research Foundation)-TRR 173268565370 (project B12).

*dpol@kth.se

[1] G. A. Prinz, Magnetoelectronics, Science 282, 1660 (1998).

[2] P. Grünberg, Layered magnetic structures: History, highlights, applications, Phys. Today 54, 5, 31 (2001).

[3] I. Žutić, J. Fabian, and S. Das Sarma, Spintronics: Fundamentals and applications, Rev. Mod. Phys. 76, 323 (2004).

[4] P. F. Carcia, A. D. Meinhaldt, and A. Suna, Perpendicular magnetic anisotropy in $\mathrm{Pd} / \mathrm{Co}$ thin film layered structures, Appl. Phys. Lett. 47, 178 (1985).

[5] W. B. Zeper, F. J. A. M. Greidanus, P. F. Carcia, and C. R. Fincher, Perpendicular magnetic anisotropy and magnetooptical Kerr effect of vapor-deposited Co/Py-layered structures, J. Appl. Phys. 65, 4971 (1989).

[6] R. Sbiaa, H. Meng, and S. N. Piramanayagam, Materials with perpendicular magnetic anisotropy for magnetic random access memory, Phys. Status Solidi RRL 5, 413 (2011).

[7] B. Dieny, Giant magnetoresistance in spin-valve multilayers, J. Magn. Magn. Mater. 136, 335 (1994).

[8] D. J. Monsma, J. C. Lodder, Th. J. A. Popma, and B. Dieny, Perpendicular Hot Electron Spin-Valve Effect in a New Magnetic Field Sensor: The Spin-Valve Transistor, Phys. Rev. Lett. 74, 5260 (1995).

[9] J. Nogués and I. K. Schuller, Exchange bias, J. Magn. Magn. Mater. 192, 203 (1999).

[10] P. Grünberg, R. Schreiber, Y. Pang, M. B. Brodsky, and H. Sowers, Layered Magnetic Structures: Evidence for Antiferromagnetic Coupling of $\mathrm{Fe}$ Layers Across $\mathrm{Cr}$ Interlayers, Phys. Rev. Lett. 57, 2442 (1986).

[11] M. N. Baibich, J. M. Broto, A. Fert, F. Nguyen Van Dau, F. Petroff, P. Etienne, G. Creuzet, A. Friederich, and J. Chazelas, Giant Magnetoresistance of $(001) \mathrm{Fe} /(001) \mathrm{Cr}$ Magnetic Superlattices, Phys. Rev. Lett. 61, 2472 (1988).

[12] G. Binasch, P. Grünberg, F. Saurenbach, and W. Zinn, Enhanced magnetoresistance in layered magnetic structures with antiferromagnetic interlayer exchange, Phys. Rev. B 39, 4828 (1989).

[13] T. Jungwirth, X. Marti, P. Wadley, and J. Wunderlich, Antiferromagnetic spintronics, Nat. Nanotechnol. 11, 231 (2016)

[14] R. A. Duine, Kyung-Jin Lee, Stuart S. P. Parkin, and M. D. Stiles, Synthetic antiferromagnetic spintronics, Nat. Phys. 14, 217 (2018).

[15] F. Y. Yang and C. L. Chien, Spiraling Spin Structure in an Exchange-Coupled Antiferromagnetic Layer, Phys. Rev. Lett. 85, 2597 (2000).

[16] D. N. H. Nam, W. Chen, K. G. West, D. M. Kirkwood, J. Lu, and S. A. Wolf, Propagation of exchange bias in CoFe/ FeMn/CoFe trilayers, Appl. Phys. Lett. 93, 152504 (2008).

[17] W. Kuch, L. I. Chelaru, F. Offi, J. Wang, M. Kotsugi, and J. Kirschner, Tuning the magnetic coupling across ultrathin antiferromagnetic films by controlling atomic-scale roughness, Nat. Mater. 5, 128 (2006). 
[18] Y. A. Shokr, M. Erkovan, O. Sandig, and W. Kuch, Temperature-induced sign change of the magnetic interlayer coupling in $\mathrm{Ni} / \mathrm{Ni}_{25} \mathrm{Mn}_{75} / \mathrm{Ni}$ trilayers on $\mathrm{Cu}_{3} \mathrm{Au}(001)$, J. Appl. Phys. 117, 175302 (2015).

[19] S. Takei, T. Moriyama, T. Ono, and Ya. Tserkovnyak, Antiferromagnet-mediated spin transfer between a metal and a ferromagnet, Phys. Rev. B 92, 020409(R) (2015).

[20] T. Hagelschuer, Y. A. Shokr, and W. Kuch, Spin-state transition in antiferromagnetic $\mathrm{Ni}_{0.4} \mathrm{Mn}_{0.6}$ films in $\mathrm{Ni} / \mathrm{NiMn} / \mathrm{Ni}$ trilayers on $\mathrm{Cu}(001)$, Phys. Rev. B 93, 054428 (2016).

[21] H. Wu, L. Huang, C. Fang, B. S. Yang, C. H. Wan, G. Q. Yu, J. F. Feng, H. X. Wei, and X. F. Han, Magnon Valve Effect Between Two Magnetic Insulators, Phys. Rev. Lett. 120, 097205 (2018).

[22] P. Tang and X. F. Han, Magnon resonant tunneling effect in double-barrier insulating magnon junctions and magnon field effect transistor, Phys. Rev. B 99, 054401 (2019).

[23] V. Baltz, A. Manchon, M. Tsoi, T. Moriyama, T. Ono, and Y. Tserkovnyak, Antiferromagnetic spintronics, Rev. Mod. Phys. 90, 015005 (2018).

[24] I. L. Prejbeanu, M Kerekes, R C Sousa, H Sibuet, O Redon, B Dieny, and J.P Nozières, Thermally assisted MRAM, J. Phys. Condens. Matter 19, 165218 (2007).

[25] A. M. Kadigrobov, S. Andersson, D. Radić, R. I. Shekhter, M. Jonson, and V. Korenivski, Thermoelectrical manipulation of nanomagnets, J. Appl. Phys. 107, 123706 (2010).

[26] A. M. Kadigrobov, S. Andersson, H. C. Park, D. Radić, R. I. Shekhter, M. Jonson, and V. Korenivski, Thermal-magneticelectric oscillator based on spin-valve effect, J. Appl. Phys. 111, 044315 (2012).

[27] D. M. Polishchuk, Yu. O. Tykhonenko-Polishchuk, A. F. Kravets, and V. Korenivski, Thermal switching of indirect interlayer exchange in magnetic multilayers, Europhys. Lett. 118, 37006 (2017).

[28] D. M. Polishchuk, Yu. O. Tykhonenko-Polishchuk, E. Holmgren, A. F. Kravets, and V. Korenivski, Thermally induced antiferromagnetic exchange in magnetic multilayers, Phys. Rev. B 96, 104427 (2017).

[29] A. F. Kravets, D. M. Polishchuk, V. A. Pashchenko, A. I. Tovstolytkin, and V. Korenivski, Current-driven thermomagnetic switching in magnetic tunnel junctions, Appl. Phys. Lett. 111, 262401 (2017).

[30] J. Torrejon, M. Riou, F. A. Araujo, S. Tsunegi, G. Khalsa, D. Querlioz, P. Bortolotti, V. Cros, K. Yakushiji, A. Fukushima, H. Kubota, S. Yuasa, M. D. Stiles, and J. Grollier, Neuromorphic computing with nanoscale spintronic oscillators, Nature (London) 547, 428 (2017).

[31] R. Cheng, D. Xiao, and J. G. Zhu, Interlayer Couplings Mediated by Antiferromagnetic Magnons, Phys. Rev. Lett. 121, 207202 (2018).
[32] R. Cheng, D. Xiao, and J.-G. Zhu, Antiferromagnet-based magnonic spin-transfer torque, Phys. Rev. B 98, 020408(R) (2018).

[33] Even though the structure is metallic, F-F* correlations across the AFM spacer due to spin-polarized conduction electrons can be neglected since the corresponding spin relaxation length in AFM is $\lesssim 1 \mathrm{~nm}$, much shorter than the AFM layer thickness range used in this work; see P. Merodio et al., Penetration depth and absorption mechanisms of spin currents in $\mathrm{Ir}_{20} \mathrm{Mn}_{80}$ and $\mathrm{Fe}_{50} \mathrm{Mn}_{50}$ polycrystalline films by ferromagnetic resonance and spin pumping, Appl. Phys. Lett. 104, 032406 (2014); J. Bass et al., CPP magnetoresistance of magnetic multilayers: A critical review, J. Magn. Magn. Mater. 408, 244 (2016).

[34] E. V. Gomonay and V. M. Loktev, Spintronics of antiferromagnetic systems (Review Article), Low Temp. Phys. 40, 17 (2014).

[35] See Supplemental Material at http://link.aps.org/ supplemental/10.1103/PhysRevLett.126.227203 for additional data and analysis, which includes Refs. [34,36-39].

[36] K. Sato, Measurement of magneto-optical Kerr effect using piezo-birefringent modulator, Jpn. J. Appl. Phys. 20, 2403 (1981).

[37] P. Q. J. Nederpel and J. W. D. Martens, Magneto-optical ellipsometer, Rev. Sci. Instrum. 56, 687 (1985).

[38] R. A. Fry, L. H. Bennett, and E. Della Torre, Reversal behavior in a bimodal magneto-optical medium, J. Vac. Sci. Techn. 17, 1331 (1999).

[39] S. Polisetty, J. Scheffler, S. Sahoo, Yi Wang, T. Mukherjee, $\mathrm{Xi} \mathrm{He}$, and $\mathrm{Ch}$. Binek, Optimization of magneto-optical kerr setup: Analyzing experimental assemblies using jones matrix formalism, Rev. Sci. Instrum. 79, 055107 (2008).

[40] F. Offi, W. Kuch, and J. Kirschner, Structural and magnetic properties of $\mathrm{Fe}_{x} \mathrm{Mn}_{1-x}$ thin films on $\mathrm{Cu}(001)$ and on Co/Cu(001), Phys. Rev. B 66, 064419 (2002).

[41] M. H. Pan, J. Chen, J. G. Long, L. N. Tong, M. Lu, J. Du, A. $\mathrm{Hu}$, and $\mathrm{H}$. R. Zhai, A $90^{\circ}$ ferromagnetic layer coupling in FM/AFM/FM structures, J. Magn. Magn. Mater. 226-230, 1817 (2001).

[42] K. Lenz, S. Zander, and W. Kuch, Magnetic Proximity Effects in Antiferromagnet/Ferromagnet Bilayers: The Impact on the Néel Temperature, Phys. Rev. Lett. 98, 237201 (2007).

[43] We discuss in detail the results for the trilayers with $t=6.0$ and $8.5 \mathrm{~nm}$, which are the most representative since their AFM-spacers are near the onset of AFM-ordering at room temperature. The results for other AFM-spacers are qualitatively same, with the thermomagnetic transition sequentially shifted higher in temperature as the AFM thickness is increased. 